\title{
Estudo de doses de NPK nas variáveis de crescimento e produtividade de inflorescências de Heliconia sp. ${ }^{1}$
}

\author{
LUCIANA BITTENCOURT FERREIRA ${ }^{2}$ e SEBASTIÃO ALBERTO OLIVEIRA ${ }^{3}$
}

\section{RESUMO}

Este trabalho teve como objetivo analisar a influência de doses de $\mathrm{N}, \mathrm{P}_{2} \mathrm{O}_{5}$ e $\mathrm{K}_{2} \mathrm{O}$ sobre as variáveis de crescimento e produtividade inicial de inflorescências de Heliconia psittacorum L. f. cv. St. Vincent Red e Heliconia psittacorum L. f. x Heliconia spathocircinata Aristeguieta 'Golden Torch', em solo de cerrado. Efetuou-se o experimento em condição de campo, na propriedade Sítio Fiore Mio, no município de Santo Antônio de Goiás (GO), em latossolo vermelho, de janeiro a outubro de 2002. Adotou-se o delineamento experimental em blocos casualizados com quinze tratamentos e três repetições, arranjados conforme matriz experimental Plan Puebla II. Os tratamentos consistiram na combinação de quatro doses de $\mathrm{N}(0,200,400 \mathrm{e}$ $600 \mathrm{~kg} / \mathrm{ha})$, quatro de $\mathrm{P}_{2} \mathrm{O}_{5}(0,80,160$ e $240 \mathrm{~kg} / \mathrm{ha})$ e quatro de $\mathrm{K}_{2} \mathrm{O}(0,80,160$ e $240 \mathrm{~kg} / \mathrm{ha})$. Aos 260 dias após o plantio, coletaram-se os dados do comprimento do pseudocaule, comprimento da haste floral e diâmetro do pedúnculo floral. A produtividade de inflorescências foi computada a cada quinze dias até 270 dias do plantio. Concluiu-se que as duas variedades responderam diferentemente à aplicação da adubação NPK. A aplicação de doses crescentes de nitrogênio favoreceu o aumento linear das quatro variáveis analisadas na Heliconia 'Golden Torch', não ocorrendo, entretanto, o mesmo para os outros dois macronutrientes. Na Heliconia 'St. Vincent Red', as doses crescentes de $\mathrm{P}_{2} \mathrm{O}_{5}$ e $\mathrm{K}_{2} \mathrm{O}$ influenciaram positi- vamente todas as variáveis analisadas e o $\mathrm{N}$ favoreceu somente o aumento linear da produção de inflorescências. As maiores produtividades de inflorescências foram obtidas no tratamento 10 (600160-160) com 17,2 inflorescências. ${ }^{-2}$ para Heliconia 'Golden Torch' e no tratamento 12 (400-240-160) com 17,1 inflorescências. ${ }^{-2}$ para Heliconia 'St. Vincent Red'.

Palavras-chave: flor de corte, NPK, inflorescências, adubação.

\section{ABSTRACT \\ Study of fertilization NPK in the growth variable and inflorescences productivity of Heliconia sp.}

This work had as objective analyzes the effect of doses of $\mathrm{N}, \mathrm{P}_{2} \mathrm{O}_{5}$ and $\mathrm{K}_{2} \mathrm{O}$ on growth variable and initial productivity of inflorescences of Heliconia psittacorum L. f. 'St. Vicent Red' and Heliconia psittacorum L. f. x Heliconia spathocircinata Aristeguieta 'Golden Torch', under savannah soil. The experiment was led in field condition on a Red Latosol, in Fiore Mio farm, at Santo Antônio de Goiás, State of Goiás, Brazil, during the period of January- October/2002. The experimental design was in randomized block, with fifteen treatments and three repetitions arranged according to experimental matrix Pan Puebla II. The treatments consisted of the combination of four

\footnotetext{
${ }^{1}$ Parte da Dissertação de Mestrado em Ciências Agrárias apresentada pela autora à Universidade de Brasília.

${ }^{2}$ Engenheira Agrônoma, Agência Goiana de Desenvolvimento Rural e Fundiário (AGÊNCIA RURAL). Departamento de Produção Vegetal. Rua Jornalista Geral Vale, 331, Setor Universitário. Caixa Postal 331. 74610-060 Goiânia (GO). E-mail: lucianadbf@terra.com.br

${ }^{3}$ Professor Adjunto, Doutor, Faculdade de Agronomia e Medicina Veterinária, Universidade de Brasília (UnB), Caixa Postal 04508. $70910-970$ Brasília (DF). E-mail: oliveira@unb.br
} 
doses of $\mathrm{N}(0,200,400$ and $600 \mathrm{~kg} / \mathrm{ha})$, four of $\mathrm{P}_{2} \mathrm{O}_{5}(0$, $80,160$ and $240 \mathrm{~kg} / \mathrm{ha})$ and four of $\mathrm{K}_{2} \mathrm{O}(0,80,160$, $240 \mathrm{~kg} / \mathrm{ha}$ ). To the 260 days after the planting was collected the data of the length of the pseudstems, length of the floral stem and diameter of the floral stalk. The inflorescences productivity was computed every fifteen days until 270 days of the planting. The obtained results allowed to conclude that the two varieties answered to the application of the differently fertilization NPK. The application of crescent doses of nitrogen to the soil favored the lineal increase of the four variables analyzed in Heliconia 'Golden Torch' not happening the same for the other two macronutrients, it was observed that the maximum doses of $\mathrm{P}_{2} \mathrm{O}_{5}$ and of $\mathrm{K}_{2} \mathrm{O}$ $(240 \mathrm{~kg} / \mathrm{ha})$ they presented decrease in the parameters analyses. In Heliconia 'St. Vicent Red', the growing doses of $\mathrm{P}_{2} \mathrm{O}_{5}$ and $\mathrm{K}_{2} \mathrm{O}$ influenced all the analyzed parameters and $\mathrm{N}$ positively it favored only the lineal increase of the inflorescences production. The largest inflorescences productivities were obtained in the treatment 10 (600-160-160) with 17.2 inflorescences. $\mathrm{m}^{-2}$ for Heliconia 'Golden Torch' and in the treatment 12 (400-240-160) with 17.1 inflorescences. ${ }^{-2}$ for Heliconia 'St Vicent Red'.

Key words: Cut flower, inflorescences, NPK, fertilization.

\section{INTRODUÇÃO}

O gênero Heliconia, pertencente à família Heliconiaceae da ordem zingiberales (MELLO FILHO \& SANTOS, 1985) tem atraído a atenção como flor de corte, tanto de produtores como de consumidores, pelo seu potencial hortícola e de ornamentação (CLEMENS \& MORTON, 1999).

Tendo como centro de origem o Norte da América do Sul, Heliconia psittacorum é uma diversificada espécie onde se estimam mais de 85 formas naturais de ocorrência, com mais de catorze variedades cultivadas comercialmente (BERRY \& KRESS, 1991), cujas principais são Andromeda, Sassy, St. Vincent Red e Tay (CASTRO \& GRAZIANO, 1997).

O híbrido natural Heliconia psittacorum L. f. x Heliconia spathocircinata Aristeguieta 'Golden Torch' - Heliconia ‘Golden Torch' está, também, entre um dos mais plantados para fins comerciais (CASTRO, 1995). Utilizando marcador molecular, KUMAR et al. (1998) relataram que há $83,8 \%$ de similaridade genética entre esse híbrido e algumas variedades de $H$. psittacorum ('Iris' e 'Petra').
O manejo usualmente empregado no Brasil favorece o aumento da produção em relação às áreas naturais, mas há carência de estudos quanto às normas técnicas de produção (IBIAPABA et al., 1997), em especial quanto a informações e padrões nutricionais (CLEMENS \& MORTON, 1999).

São plantas adaptadas a solos ácidos, com $\mathrm{pH}$ na faixa de 4,5 a 6,5 (CASTRO, 1995), bem drenados (CRILEY, 1989) e ricos em matéria orgânica (BERRY \& KRESS, 1991).

Trabalhando com plantas de Heliconia 'Golden Torch', JIE et al. (2000) analisaram características relacionadas com fotossíntese e com conteúdo foliar de nitrogênio, demonstrando que houve correlação positiva entre eles quando cultivados a pleno sol e com fornecimento de altos níveis do nutriente.

Conforme relatado por CASTRO (1995), os nutrientes mais exigidos por esta cultura são nitrogênio, potássio, fósforo, magnésio, ferro e manganês, e que adubações parceladas em duas a três vezes por ano, na proporção de 3:1:2 de $\mathrm{N}, \mathrm{P}$ e K resultam num rápido crescimento e florescimento. BERRY \& KRESS (1991) citam o uso da razão $1 \mathrm{~N}$ :2K para $H$. psittacorum em plantio na Holanda.

Segundo CRILEY (1989), produtores havaianos utilizam um programa de adubação que inclui a aplicação de $200 \mathrm{~g}$ de adubo mineral contendo $\mathrm{N}-\mathrm{P}_{2} \mathrm{O}_{5}-\mathrm{K}_{2} \mathrm{O}$ na relação $1: 1: 1$, de três a quatro aplicações por ano por planta, obtendo resultados satisfatórios em suas produções, apesar da não-comprovação científica.

No Estado de São Paulo, LOPES \& GRAZIANO (2001) relatam a ausência de critérios para a aplicação da adubação de cobertura, ocorrendo desde a aplicação quinzenal de NPK (6-18-18) até adubações duas vezes por ano da fórmula 10-10-10.

Ao trabalhar com Heliconia 'Golden Torch' plantadas em vasos, CLEMENS \& MORTON (1999) observaram que, dos tratamentos utilizados, a aplicação de $1,2 \mathrm{~kg}$ de N$/ \mathrm{m}^{3}$ de terra e também a relação 1,2 $\mathrm{kg}$ de $\mathrm{N}$ com $0,6 \mathrm{~kg}$ de $\mathrm{K}_{2} \mathrm{O} / \mathrm{m}^{3}$ de terra, favoreceram o aumento da produção de biomassa, com reflexos no crescimento vegetativo e no tamanho de flores e que com a aplicação de doses menores dos mesmos nutrientes obtiveram maior produção de inflorescências.

A região dos cerrados, localizada nos trópicos, reúne características favoráveis às diversas atividades agropecuárias, devendo-se estudar, avaliar e desenvolver tecnologias que potencializem seu aproveitamento (WAGNER, 1986). De acordo com SÁNCHEZ \& 
SALINAS (1981), as deficiências mais acentuadas nesses solos são de nitrogênio, fósforo e potássio. Apresentam fixação do fósforo, deficiência de enxofre, zinco, cálcio e magnésio, e elevada saturação de alumínio e acidez. Assim, há necessidade de informações sobre a adequação de espécies às características de cada nova região de produção, como é o caso do cerrado goiano, evitando que produtores utilizem tecnologias oriundas de outras regiões, limitando sua potencialidade e exploração econômica.

Este trabalho, portanto, teve por objetivo analisar a influência de doses de $\mathrm{N}, \mathrm{P}_{2} \mathrm{O}_{5}$ e $\mathrm{K}_{2} \mathrm{O}$ sobre variáveis de crescimento e produtividade inicial de inflorescências de duas variedades de helicônias, em solo do cerrado.

\section{MATERIAL E MÉTODOS}

Desenvolveu-se o experimento em condição de campo, na propriedade Sítio Fiore Mio, localizada no município de Santo Antônio de Goiás (GO), distante $30 \mathrm{~km}$ do centro da cidade de Goiânia, de janeiro a outubro de 2002. A altitude média é de $823 \mathrm{~m}$, latitude de $16^{\circ} 28^{\prime} 00$ " S e longitude de $49^{\circ} 17^{\prime} 00^{\prime}$ ' W. O clima do local, segundo o Sistema Internacional de Köppen, é classificado como Tropical Estacional - Aw, apresentando verão chuvoso e inverno seco. O solo predominante é o latossolo vermelho-escuro e, de acordo com a nova nomenclatura brasileira para solos como latossolo vermelho, estava revestido com capim-braquiária (Brachiaria decumbens Stapt.), porém sem haver manejo de animais.

Para a obtenção das plantas, selecionaram-se propágulos vegetativos (rizomas) de Heliconia psittacorum cv. St. Vicent Red e de Heliconia psittacorum L. f. x Heliconia spathocircinata
Aristeguieta 'Golden Torch', oriundos de plantas matrizes com mais de dois anos de idade, provenientes de plantio comercial existente na propriedade. As touceiras foram individualizadas em porções de rizomas com, aproximadamente, $10 \mathrm{~cm}$ e segmento do pseudocaule com mais de $25 \mathrm{~cm}$ de comprimento. Após limpeza para remoção do solo, folhas secas e excesso de raízes, efetuou-se tratamento fitossanitario (CASTRO, 1995), cobrindo-se com palhada de capim, por 24 horas, a fim de estimular o intumescimento inicial das gemas para o desenvolvimento das novas brotações (IBIAPABA et al., 1997). Os rizomas foram plantados em sacos de polietileno de $17 \times 22 \mathrm{~cm}$, com substrato preparado na propriedade (mistura de resíduo vegetal carbonizado e terra de barranco) e mantidos à meia-sombra com irrigação constante por cinco meses (agosto a dezembro de 2001) adquirindo porte suficiente para serem transplantados ao campo.

As propriedades químicas e características físicas das amostras compostas da área do experimento, coletadas a $20 \mathrm{~cm}$ de profundidade encontram-se na Tabela 1.

Fez-se o controle inicial do capim-braquiária mediante a aplicação de herbicida específico e incorporação dos restos vegetais, e capinas periódicas até o final do experimento.

Os tratamentos foram arranjados de acordo com matriz experimental Plan Puebla II, conforme FERNANDEZ \& LAIRD (1978), sendo o delineamento experimental em blocos casualizados, com quinze tratamentos e três repetições.

As doses de cada nutriente e as fontes utilizadas foram as seguintes: $\mathrm{N}: 0,200,400$ e $600 \mathrm{~kg} / \mathrm{ha}$, via sulfato de amônio; $\mathrm{P}_{2} \mathrm{O}_{5}: 0,80,160$ e $240 \mathrm{~kg} / \mathrm{ha}$, via termofosfato Yoorin; $\mathrm{K}_{2} \mathrm{O}: 0,80,160$ e $240 \mathrm{~kg} / \mathrm{ha}$, via cloreto de potássio.

Tabela 1. Propriedades químicas e características físicas de amostras da camada de $0-20 \mathrm{~cm}$ do solo da área do experimento, colhidas antes e dez meses após a aplicação do calcário

\begin{tabular}{|c|c|c|c|c|c|c|c|c|c|c|c|c|c|c|c|c|c|c|c|c|c|c|c|}
\hline Solo & $\mathrm{pH}$ & $\mathrm{pH}$ & M.O. & $\mathrm{P}$ & K & $\mathrm{S}$ & $\mathrm{Ca}$ & $\mathrm{Mg}$ & $\mathrm{Al}$ & $\mathrm{H}+\mathrm{A}$ & CTC & $\mathrm{Ca}$ & $\mathrm{Mg}$ & K & V & B & $\mathrm{Cu}$ & $\mathrm{Fe}$ & $\mathrm{Mn}$ & $\mathrm{Zn}$ & Areia & Silte & Argila \\
\hline & Água & $\begin{array}{l}\mathrm{CaCl}_{2} \\
0,01 \mathrm{M}\end{array}$ & $\begin{array}{l}\mathrm{dag} / \\
\mathrm{dm}^{3}\end{array}$ & $\mathrm{n}$ & $\mathrm{g} / \mathrm{dr}$ & 3 & 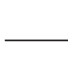 & & mo & $\mathrm{dm}^{3}-$ & & & $\%$ & & & - & & $\mathrm{mg}$ & & & & $\%$ & \\
\hline 1 & 6,0 & 5,0 & 3,5 & 1,0 & 116 & 5,3 & 1,9 & 0,9 & 0, & 4, & , & 2 & 11 & 4 & 39 & 0,2 & 1,5 & 72 & 71 & 1,1 & 33 & 17 & 50 \\
\hline 2 & 6,4 & 5,5 & 3,5 & 1,5 & 86 & 6,5 & 2,9 & 1,4 & 0,0 & 3,5 & 8,0 & 36 & 18 & $J$ & 56 & 0,5 & 1,4 & 62 & 68,4 & 4,4 & 35 & 17 & 48 \\
\hline
\end{tabular}

Obs.: Solo 1: Análise do solo antes da aplicação de calcário.

Solo 2: Análise do solo após dez meses da aplicação de calcário. 
A parcela experimental constituiu-se de três linhas com cinco covas cada uma, formando um total de quinze covas por parcela $\left(9,0 \mathrm{~m}^{2}\right)$, sendo a área útil as covas centrais $\left(1,8 \mathrm{~m}^{2}\right)$. As covas, com diâmetro de $30 \times 30 \mathrm{x}$ $30 \mathrm{~cm}$, foram abertas num espaçamento de $0,5 \mathrm{~m}$ entre plantas e 1,2 m entre linhas.

Aplicou-se o fertilizante fosfatado nas covas de plantio, de acordo com as quantidades definidas em cada tratamento, parcelando-se a adubação nitrogenada e potássica em três aplicações iguais e distribuídas a lanço, aos 30, 90 e 150 dias após o plantio. Os micronutrientes, na forma de fritas (FTE BR-12), foram adicionados em quantidades iguais para todos os tratamentos, na proporção de $50 \mathrm{~kg} /$ ha ou $5 \mathrm{~g} / \mathrm{m}^{2}$, junto à segunda aplicação da adubação de cobertura, aos 90 dias do plantio.

$\mathrm{Na}$ calagem, aplicou-se 0,93 t/ha de calcário dolomítico (CaO: 30\%, MgO: 18\% e PRNT =95\%), a qual foi calculada pela fórmula de saturação por bases, para $\mathrm{V}_{2}=50 \%$, de acordo com RAIJ et al. (1987).

Irrigou-se, por meio de aspersão convencional, durante todo o período da seca, com uma lâmina líquida de, aproximadamente, $5 \mathrm{~mm} / \mathrm{hora} / \mathrm{dia}$, a cada dois dias.

Coletaram-se os dados das variáveis aos 260 dias do plantio definitivo, tomando-se dez plantas por parcela, escolhidas ao acaso, avaliando-se:

- Comprimento do pseudocaule: tomado do colo da planta até a inserção da folha mais nova.

- Comprimento da haste floral: colo da planta até a base da inflorescência.

- Diâmetro do pedúnculo floral: base do pedúnculo floral, na altura da inserção da folha mais nova.

- Produtividade de inflorescências: a contagem iniciou-se quando as primeiras inflorescências estavam com, pelo menos, uma bráctea aberta. Ocorreu a cada quinze dias, estendendo-se até a primeira quinzena de outubro de 2002, totalizando 270 dias desde o plantio.

Os resultados dos dados coletados foram submetidos à análise da variância, considerando-se o delineamento estatístico de blocos casualizados. Utilizou-se o teste de significância $\mathrm{F}$ para $1 \%$ e $5 \%$ de probabilidade, sendo as médias comparadas pelo teste de Tukey, também a 5\% de probabilidade, e submetidos à analise da regressão, com a finalidade de determinar a relação existente entre a variável e a dose do nutriente aplicada (PIMENTEL-GOMES, 1985).

\section{RESULTADOS E DISCUSSÃO}

\subsection{Comprimento do pseudocaule}

Pela análise de regressão, pode-se verificar que o efeito das doses de nitrogênio para Heliconia 'Golden Torch' permitiram uma equação de regressão linear significativa $\left(\mathrm{y}=34,288+0,014 \mathrm{x} \mathrm{R}^{2}=0,92 *\right)$, ou seja, o comprimento do pseudocaule aumentou em relação às doses crescentes do nutriente em estudo. Já para Heliconia 'St. Vincent Red', as doses de N não apresentaram resposta significativa para a variável analisada $\left(\mathrm{y}=46,293-0,005 \mathrm{x} \quad \mathrm{R}^{2}=0,3461^{\mathrm{ns}}\right)$.

Na presença da adubação fosfatada, a análise de regressão mostrou-se significativa para as duas variedades. Para Heliconia 'Golden Torch' a equação quadrática foi significativa $(\mathrm{y}=31,699+0,1445 \mathrm{x}-$ $0,0006 x^{2} R^{2}=0,99^{*}$ ), sendo o maior comprimento médio do pseudocaule, $45,1 \mathrm{~cm}$, obtido utilizando $160 \mathrm{~kg} /$ ha de $\mathrm{P}_{2} \mathrm{O}_{5}$. Para Heliconia 'St. Vincent Red', a equação significativa foi a linear $\left(\mathrm{y}=41,827+0,0427 \mathrm{x} \mathrm{R}^{2}\right.$ $\left.=0,77^{*}\right)$, indicando que aumentos nas doses de fósforo favoreceram o aumento no comprimento dos pseudocaules.

Na adubação potássica, a análise de regressão para Heliconia 'Golden Torch' apresentou efeito quadrático significativo $\left(\mathrm{y}=31,882+0,1403 \mathrm{x}-0,0006 \mathrm{x}^{2} \mathrm{R}^{2}=\right.$ $0,99 * *$ ), cujo comprimento máximo ocorreu com a aplicação de $80 \mathrm{~kg} / \mathrm{ha}$ de $\mathrm{K}_{2} \mathrm{O}$. Apesar de as doses de potássio não terem apresentado resposta significativa para Heliconia 'St. Vincent Red', a curva de regressão identificou tendência de aumento no comprimento do pseudocaule com o incremento de $\mathrm{K}_{2} \mathrm{O}(\mathrm{y}=42,041+$ $\left.0,0234 \times \mathrm{R}^{2}=0,56^{\mathrm{ns}}\right)$.

A analise da variância demonstrou que houve diferença estatisticamente significativa entre elas $(p<$ $0,05)$. Durante a época avaliada, a média total do comprimento do pseudocaule de Heliconia 'St. Vincent Red' foi maior do que Heliconia 'Golden Torch', 45,5 e 38,3 $\mathrm{cm}$ respectivamente (Tabela 2 ).

\subsection{Comprimento da haste floral}

Para Heliconia 'St. Vincent Red', as curvas de regressão para $\mathrm{P}_{2} \mathrm{O}_{5}\left(\mathrm{y}=70,93-0,0421 \mathrm{x}+0,0004 \mathrm{x}^{2}\right.$ $\left.\mathrm{R}^{2}=0,99^{\text {ns }}\right)$ e $\mathrm{K}_{2} \mathrm{O}\left(\mathrm{y}=67,712+0,0378 \mathrm{x} \mathrm{R}^{2}=0,70^{\text {ns }}\right)$ indicam que o acréscimo das doses mostrou tendência de aumento nos comprimentos das hastes florais, o que não aconteceu para $\mathrm{N}(\mathrm{y}=69,7111+0,0324 \mathrm{x}-$ $\left.6 \mathrm{E}-05 \mathrm{x}^{2} \mathrm{R}^{2}=0,7262^{\mathrm{ns}}\right)$. 
$\mathrm{Na}$ análise da regressão para adubação nitrogenada em Heliconia 'Golden Torch', a resposta teve efeito linear significativo $\left(\mathrm{y}=45,851+0,0251 \times \mathrm{R}^{2}=0,95^{* *}\right)$, indicando que o aumento das doses de $\mathrm{N}$ favoreceu $\mathrm{o}$ do comprimento da haste floral. A adubação fosfatada não mostrou significância, apesar de a curva de regressão mostrar que a dose de $240 \mathrm{~kg} \mathrm{P}_{2} \mathrm{O}_{5} /$ ha tendeu a uma redução no comprimento da haste floral $(\mathrm{y}=43,241+$ $\left.0,1962 x-0,0007 x^{2} R^{2}=0,99^{n s}\right)$. A ação das doses de $\mathrm{K}_{2} \mathrm{O}$ teve como resposta significativa uma equação de efeito quadrático $\left(\mathrm{y}=43,704+0,1915 \mathrm{x}+0,0007 \mathrm{x}^{2} \mathrm{R}^{2}\right.$ $\left.=0,99^{*}\right)$, onde o tratamento $7\left(400 \mathrm{~N}-160 \mathrm{P}_{2} \mathrm{O}_{5}-80\right.$ $\mathrm{K}_{2} \mathrm{O}$ ) apresentou o maior comprimento de haste floral, com $61,6 \mathrm{~cm}$, utilizando a dosagem de $80 \mathrm{~kg} \mathrm{~K}_{2} \mathrm{O} / \mathrm{ha}$.

$\mathrm{Na}$ analise da variância entre as duas variedades, observou-se que houve diferença estatisticamente significativa $(\mathrm{p}<0,01)$ entre ambas, indicando que as duas variedades responderam diferentemente às doses de NPK aplicadas, apresentando Heliconia 'St. Vincent Red' os maiores comprimentos da haste floral (Tabela 2).

\subsection{Diâmetro do pedúnculo floral}

A análise da regressão, em função das doses de nitrogênio não mostrou significância para nenhuma das duas variedades. Entretanto, na Heliconia 'Golden Torch', com a aplicação de doses crescentes de N, houve uma tendência de aumento no diâmetro dos pedúnculos florais $\left(\mathrm{y}=0,5092+9 \mathrm{E}-05 \mathrm{x} \mathrm{R}^{2}=0,94^{\mathrm{ns}}\right)$. Na Heliconia 'St. Vincent Red', o N praticamente não causou alterações nesta variável $(\mathrm{y}=0,3842+3 \mathrm{E}-07 \mathrm{x}$ $\left.\mathrm{R}^{2}=0,00^{\mathrm{ns}}\right)$

A adubação fosfatada, na Heliconia 'St. Vincent Red', a análise da regressão apresentou significância com efeito quadrático $\left(\mathrm{y}=0,3829-0,0002 \mathrm{x}+2 \mathrm{E}-06 \mathrm{x}^{2}\right.$ $\mathrm{R}^{2}=0,99 *$ ), indicando que o acréscimo na dose de $\mathrm{P}_{2} \mathrm{O}_{5}$ favoreceu o aumento do diâmetro do pedúnculo floral. Já na Heliconia 'Golden Torch', a adição crescente de fósforo ao solo, apesar de não significativa estatisticamente, apresentou tendência de aumento linear nessa variável $\left(\mathrm{y}=0,5099+0,0002 \mathrm{x} \quad \mathrm{R}^{2}=0,87^{\mathrm{ns}}\right)$.

Tabela 2. Análise da variância para as variáveis: comprimento do pseudocaule $(\mathrm{cm})$, comprimento de haste floral $(\mathrm{cm})$, diâmetro do pedúnculo floral $(\mathrm{cm})$ na altura da folha mais nova, aos 260 dias do plantio, e produtividade de inflorescências. $\mathrm{m}^{-2}$ aos 270 dias do plantio, em função de doses de $\mathrm{N}, \mathrm{P}_{2} \mathrm{O}_{5}$ e $\mathrm{K}_{2} \mathrm{O}$. Média de três repetições

\begin{tabular}{|c|c|c|c|c|c|c|c|c|}
\hline \multirow{2}{*}{$\frac{\text { Tratamentos }}{\mathrm{N}-\mathrm{P}_{2} \mathrm{O}_{5}-\mathrm{K}_{2} \mathrm{O}}$} & \multicolumn{2}{|c|}{$\begin{array}{l}\text { Comprimento } \\
\text { pseudocaule }\end{array}$} & \multicolumn{2}{|c|}{$\begin{array}{l}\text { Comprimento haste } \\
\text { floral }\end{array}$} & \multicolumn{2}{|c|}{$\begin{array}{l}\text { Diâmetro pedúnculo } \\
\text { floral }\end{array}$} & \multicolumn{2}{|c|}{ Inflorescência } \\
\hline & $\begin{array}{l}\text { H. 'Golden } \\
\text { Torch' }\end{array}$ & $\begin{array}{l}H . \text { 'St. } \\
\text { Vincent } \\
\text { Red' }\end{array}$ & $\begin{array}{l}H . \text { 'Golden } \\
\text { Torch' }\end{array}$ & $\begin{array}{l}H . \text { 'St. } \\
\text { Vincent } \\
\text { Red' }\end{array}$ & $\begin{array}{l}\text { H. 'Golden } \\
\text { Torch' }\end{array}$ & $\begin{array}{l}H . \text { 'St. } \\
\text { Vincent } \\
\text { Red' }\end{array}$ & $\begin{array}{l}\text { H. 'Golden } \\
\text { Torch' }\end{array}$ & $\begin{array}{l}H . \text { 'St. } \\
\text { Vincent } \\
\text { Red' }\end{array}$ \\
\hline & & & 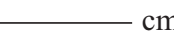 & & & & $-\mathrm{u}$ & 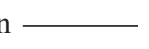 \\
\hline 1: $200-80-80$ & $41,3 \mathrm{ab}$ & $47,6 \mathrm{a}$ & $54,1 \mathrm{a}$ & $73,3 \mathrm{a}$ & $0,51 \mathrm{a}$ & $0,38 \mathrm{a}$ & $9,3 \mathrm{ab}$ & $14,3 \mathrm{a}$ \\
\hline $2: 200-80-160$ & $40,5 \mathrm{ab}$ & $39,3 \mathrm{a}$ & $57,7 \mathrm{a}$ & $64,4 \mathrm{a}$ & $0,57 \mathrm{a}$ & $0,37 \mathrm{a}$ & $10,4 \mathrm{ab}$ & $11,1 \mathrm{a}$ \\
\hline $3: 200-160-80$ & $39,4 \mathrm{ab}$ & $46,2 \mathrm{a}$ & $53,8 \mathrm{a}$ & $76,1 \mathrm{a}$ & $0,51 \mathrm{a}$ & $0,39 \mathrm{a}$ & $11,9 \mathrm{ab}$ & $15,0 \mathrm{a}$ \\
\hline $4: 200-160-160$ & $40,5 \mathrm{ab}$ & $50,2 \mathrm{a}$ & $55,2 \mathrm{a}$ & $79,2 \mathrm{a}$ & $0,53 \mathrm{a}$ & $0,42 \mathrm{a}$ & $11,7 \mathrm{ab}$ & $13,2 \mathrm{a}$ \\
\hline $5: 400-80-80$ & $40,9 \mathrm{ab}$ & $47,2 \mathrm{a}$ & $57,2 \mathrm{a}$ & $75,3 \mathrm{a}$ & $0,55 \mathrm{a}$ & $0,39 \mathrm{a}$ & $12,1 \mathrm{ab}$ & $12,8 \mathrm{a}$ \\
\hline $6: 400-80-160$ & $40,2 \mathrm{ab}$ & $44,8 \mathrm{a}$ & $55,8 \mathrm{a}$ & $73,5 \mathrm{a}$ & $0,52 \mathrm{a}$ & $0,38 \mathrm{a}$ & $9,3 \mathrm{ab}$ & $16,3 \mathrm{a}$ \\
\hline $7: 400-160-80$ & $45,1 \mathrm{a}$ & $44,4 \mathrm{a}$ & $61,6 \mathrm{a}$ & $71,6 \mathrm{a}$ & $0,54 \mathrm{a}$ & $0,40 \mathrm{a}$ & $14,1 \mathrm{ab}$ & $14,3 \mathrm{a}$ \\
\hline $8: 400-160-160$ & $40,6 \mathrm{ab}$ & $43,7 \mathrm{a}$ & 56,6 a & $72,6 \mathrm{a}$ & $0,52 \mathrm{a}$ & $0,38 \mathrm{a}$ & $15,6 \mathrm{ab}$ & $13,0 \mathrm{a}$ \\
\hline $9: 0-80-80$ & $38,6 \mathrm{ab}$ & $50,8 \mathrm{a}$ & $50,7 \mathrm{a}$ & $74,3 \mathrm{a}$ & $0,54 \mathrm{a}$ & $0,41 \mathrm{a}$ & $6,9 \mathrm{~b}$ & $14,1 \mathrm{a}$ \\
\hline 10: $600-160-160$ & $42,8 \mathrm{a}$ & $41,8 \mathrm{a}$ & $61,4 \mathrm{a}$ & $68,2 \mathrm{a}$ & $0,57 \mathrm{a}$ & $0,38 \mathrm{a}$ & $17,2 \mathrm{a}$ & $15,6 \mathrm{a}$ \\
\hline 11: $200-0-80$ & $34,9 \mathrm{ab}$ & $45,4 \mathrm{a}$ & $46,9 \mathrm{a}$ & $75,0 \mathrm{a}$ & $0,54 \mathrm{a}$ & $0,42 \mathrm{a}$ & $13,4 \mathrm{ab}$ & $13,2 \mathrm{a}$ \\
\hline $12: 400-240-160$ & $33,3 \mathrm{ab}$ & $54,3 \mathrm{a}$ & $47,5 \mathrm{a}$ & $84,4 \mathrm{a}$ & $0,57 \mathrm{a}$ & $0,43 \mathrm{a}$ & $10,6 \mathrm{ab}$ & $17,1 \mathrm{a}$ \\
\hline $13: 200-80-0$ & $34,8 \mathrm{ab}$ & $39,2 \mathrm{a}$ & $47,8 \mathrm{a}$ & $64,3 \mathrm{a}$ & $0,50 \mathrm{a}$ & $0,35 \mathrm{a}$ & $10,4 \mathrm{ab}$ & $11,1 \mathrm{a}$ \\
\hline $14: 400-160-240$ & $33,7 \mathrm{ab}$ & $46,7 \mathrm{a}$ & $47,1 \mathrm{a}$ & $75,6 \mathrm{a}$ & $0,52 \mathrm{a}$ & $0,40 \mathrm{a}$ & $10,8 \mathrm{ab}$ & $16,3 \mathrm{a}$ \\
\hline $15: 0-0-0$ & $28,6 \mathrm{~b}$ & $40,9 \mathrm{a}$ & $39,7 \mathrm{a}$ & $66,4 \mathrm{a}$ & $0,48 \mathrm{a}$ & $0,35 \mathrm{a}$ & $7,2 \mathrm{~b}$ & $9,3 \mathrm{a}$ \\
\hline Média & 38,3 & 45,5 & 52,9 & 73,3 & 0,53 & 0,39 & 11,4 & 13,8 \\
\hline $\mathrm{F}$ & $2,62 *$ & $1,28 \mathrm{~ns}$ & 1,60 ns & $1,06^{\mathrm{ns}}$ & $1,38^{\mathrm{ns}}$ & $1,05^{\mathrm{ns}}$ & $2,47 *$ & $1,59^{\mathrm{ns}}$ \\
\hline $\mathrm{CV} \%$ & 6,2 & 7,1 & 8,1 & 6,4 & 1,9 & 2,2 & 16,2 & 11,6 \\
\hline
\end{tabular}

Valores com letras iguais não diferiram significativamente pelo teste de Tukey ao nível de 5\%.

* significativo a $5 \%$ de probabilidade pelo teste $\mathrm{F}$; ns - não significativo. 
Com relação à adubação potássica, a análise da regressão não apresentou efeito significativo para Heliconia 'St. Vincent Red', porém notou-se tendência de acréscimo no diâmetro do pedúnculo floral quando se aumentou a dose de $\mathrm{K}_{2} \mathrm{O}\left(\mathrm{y}=0,3624+0,0002 \mathrm{x}^{2}=\right.$ $\left.0,54^{\mathrm{ns}}\right)$. Na Heliconia 'Golden Torch', as doses de $\mathrm{K}_{2} \mathrm{O}$ tiveram efeito quadrático $(\mathrm{y}=0,4891+0,0008 \mathrm{x}-$ $\left.3 \mathrm{E}-06 \mathrm{x}^{2} \mathrm{R}^{2}=0,99^{*}\right)$, sendo o maior diâmetro $(0,57 \mathrm{~cm})$ obtido com a dosagem de $160 \mathrm{~kg} / \mathrm{ha}$ de $\mathrm{K}_{2} \mathrm{O}$.

Os valores de todos os tratamentos de Heliconia 'Golden Torch' foram superiores aos encontrados para Heliconia 'St. Vincent Red', o que é comprovado pela análise da variância $(\mathrm{p}<0,01)$ entre as duas variedades (Tabela 2).

\subsection{Produtividade de inflorescências}

Nas duas variedades, observou-se o surgimento de botões florais a partir de 135 dias, com as primeiras inflorescências abrindo por volta de 150 dias do plantio, momento em que se iniciou sua contagem. Observou-se, em todos os tratamentos, que a floração não sofreu interrupções durante todo o período avaliado, pois quando não havia inflorescências abertas no momento da contagem, existia a presença de botões florais. O período total da avaliação coincidiu com a denominada estação seca da região dos cerrados.

Tanto na Heliconia 'St. Vincent Red' (y = $\left.11,735+0,0068 \mathrm{x} \mathrm{R}^{2}=0,97^{* *}\right)$ como na Heliconia 'Golden Torch' ( $\left.\mathrm{y}=7,142+0,0157 \mathrm{x} \mathrm{R} \mathrm{R}^{2}=0,94^{* *}\right)$ as equações de regressão foram significativas para doses de N, com efeito linear, indicando que as variedades responderam à adição de nitrogênio ao solo com aumento no número de inflorescências. $\mathrm{m}^{-2}$.

A adubação fosfatada, em Heliconia 'St. Vincent Red' apresentou equação linear significativa ( $\mathrm{y}=11,198$ $+0,0235 \times \mathrm{R}^{2}=0,98^{* *}$ ), proporcionando, com o aumento das doses de $\mathrm{P}_{2} \mathrm{O}_{5}$ elevacão o número de inflorescências produzidas por $\mathrm{m}^{2}$. Na Heliconia 'Golden Torch', a equação de regressão não apresentou efeito significativo estatisticamente $\left(\mathrm{y}=10,312+0,0058 \mathrm{x} \mathrm{R}^{2}=\right.$ $\left.0,12^{\text {ns }}\right)$, não havendo resposta satisfatória para incrementos na quantidade de inflorescências. $\mathrm{m}^{-2}$.

De acordo com os resultados obtidos para Heliconia 'St. Vincent Red', a adição de doses de potássio proporcionou efeito significativo linear para número de inflorescências. $\mathrm{m}^{-2}(\mathrm{y}=10,873+0,0235 \mathrm{x}$ $\left.\mathrm{R}^{2}=0,90^{* *}\right)$. Em Heliconia 'Golden Torch', a análise de regressão não apresentou significância, estatisticamente, porém a curva de regressão indicou uma equação quadrática $\left(\mathrm{y}=8,713+0,0476 \mathrm{x}-0,0002 \mathrm{x}^{2} \mathrm{R}^{2}=\right.$ $\left.0,98^{\text {ns }}\right)$, com a dose de $160 \mathrm{~kg} /$ ha apresentando a maior produtividade, 17,2 inflorescências. $\mathrm{m}^{-2}$.

A análise da variância entre as variedades apresentou diferença significativa $(\mathrm{p}<0,01)$, ou seja, responderam diferentemente às doses de $\mathrm{N}, \mathrm{P}_{2} \mathrm{O}_{5}$ e $\mathrm{K}_{2} \mathrm{O}$, durante o período avaliado. Observou-se, ainda, que Heliconia 'St. Vincent Red' foi mais produtiva que Heliconia 'Golden Torch': sua média total foi de 13,8 inflorescências.m ${ }^{-2}$ e Heliconia 'Golden Torch', de 11,4 inflorescências. $\mathrm{m}^{-2}$.

\section{CONCLUSÕES}

1. As duas variedades responderam diferentemente à aplicação da adubação NPK, tanto nas variáveis de crescimento quanto na produção de inflorescências.

2. Na Heliconia 'Golden Torch', o N favoreceu o aumento do comprimento dos pseudocaules, das hastes florais e do diâmetro do pedúnculo floral, além do aumento linear na produtividade de inflorescências.

3. A aplicação de $240 \mathrm{~kg} \mathrm{P}_{2} \mathrm{O}_{5} /$ ha na Heliconia 'Golden Torch' apresentou tendência de diminuição no comprimento dos pseudocaules e da haste floral.

4. A utilização de $240 \mathrm{~kg} \mathrm{~K}_{2} \mathrm{O} /$ ha na Heliconia 'Golden Torch' causou diminuição no comprimento dos pseudocaules, comprimento da haste floral, diâmetro dos pedúnculos florais e na produtividade de inflorescências.

5. O fósforo e o potássio influenciaram positivamente o aumento do comprimento dos pseudocaules, das hastes florais e do diâmetro dos pedúnculos florais, na Heliconia 'St. Vincent Red'.

6. As doses crescentes de $\mathrm{N}, \mathrm{P}_{2} \mathrm{O}_{5}$ e $\mathrm{K}_{2} \mathrm{O}$ proporcionaram aumento linear na produção de inflorescências na Heliconia 'St. Vincent Red'.

\section{REFERÊNCIAS BIBLIOGRÁFICAS}

BERRY, F. \& KRESS, W.J. Heliconia: an identification guide. Honk Kong: Smithsonian Institution, 1991. 334p.

CASTRO, C.E.F. de. Helicônia para exportação: aspectos técnicos de produção. Brasília: MAARA/SDR/ FRUPEX/SPI, 1995. 44p. (Publicações Técnicas, 16.) 
CASTRO, C.E.F. de \& GRAZIANO, T.T. Espécies do gênero Heliconia (Heliconiaceae) no Brasil. Rev. Bras. Hortic. Ornam., v.3, n.2, p.15-28, 1997.

CLEMENS, J. \& MORTON, R.H. Optimizing mineral nutrition for flower production in Heliconia 'Golden Torch' using response surface methodology. J. Amer. Soc. Hort. Sci., v.124, n.6, p.713-718, 1999.

CRILEY, R.A. Development of heliconia and alpinia in Hawaii: cultivar selection and culture. Acta Horticulturae, n. 246, p.247-258, 1989.

FERNANDEZ, A.T. \& LAIRD, R.J. La matriz experimental Plan Puebla, para ensayos sobre prácticas de producción de cultivos. 3 . ed. Chapingo, México: Colégio de Postgraduados de la Escuela Nacional de Agricultura, S. A. G., 1978. 27p.

HAAG, H.P.A nutrição mineral e o ecossistema. In: CASTRO, P.C.R.; FERREIRA, S.O. \& YAMADA,T. (Eds.).Ecofisiologia da produção agrícola. Piracicaba: Potafos, 1987. p.49-69.

IBIAPABA, M.V.B. da; LUZ, J.M.Q. \& INNECCO, R. Comportamento de duas espécies de helicônias em diferentes espaçamentos de plantio em Fortaleza (CE). Rev. Bras. Hortic. Ornam., v.3, n.2, p.74-79, 1997.

JIE, H.; LAY, P.T. \& CHONG, J.G. Alleviation of photoinhibition in Heliconia grown under tropical natu- ral conditions after release from nutrient stress. Journal of Plant Nutrition, v.23, n.2, p.181-196, 2000. (Abstract.)

KUMAR, P.P.; YAU, J.C.K. \& GOH, C.J. Genetic analyses of Heliconia species and cultivars with randomly amplified polymorphic DNA (RAPD) markers. J. Amer. Soc. Hort. Sci., v.123, n.1, p.91-97, 1998.

LOPES, C.S. \& GRAZIANO, T.T. A produção e a comercialização de Heliconia sp. no Estado de São Paulo. Rev. Bras. Hortic. Ornam., v.7, n.2, p.81-88, 2001.

MELLO FILHO, L.E. de \& SANTOS, E. Heliconiaceas. Flora Ilustrada Catarinense, 1985. p.3-13.

PIMENTEL-GOMES, F.P. Curso de estatística experimental. 11.ed. Piracicaba: Nobel. 1985. 466p.

RAIJ, B. van et al. Análise química do solo para fins de fertilidade. Campinas: Fundação Cargill, 1987. 170p.

SÁNCHEZ, P.A. \& SALINAS, J.G. Low-input technology for managing oxisols and ultisols in tropical América. Advances in Agronomy, v.34, p.279-406, 1981.

WAGNER, E. Desenvolvimento da região dos cerrados. In: GOEDERT, W.J. (ed.). Solos dos Cerrados: tecnologias e estratégias de manejo. São Paulo: Nobel, 1986. p.33-74. 